uncertain margins was to cheque the site at 3 months and 12 months post index therapeutic procedure.

Results LCSP accounted for $3.42 \%$ of adenomas, mean size $32.2 \mathrm{~mm}$ (1.02\% were LSCP > 40mm). 33/40 (82.5\%) LCSP were managed endoscopically, with either enbloc EMR, piecemeal EMR, or laparoscopically-assisted EMR. Of these, $84.8 \%$ had successful endoscopic resection with no recurrence at 3 to 12 months. Recurrent or residual polyp was detected in $1 / 28$ (3.6\%) at 3 months, with no recurrence at 12 months. $4 / 29(13.8 \%)$ of lesions initially managed endoscopically subsequently required surgery. $3 / 4$ (75\%) went on to undergo TEM and $1 / 4(25 \%)$ a segmental colonic resection. Indications included technical limitations to endoscopic management; difficult access or previous attempts at endoscopic resection at a different centre. No significant adverse events occured in the endoscopically managed group. There was a cancer rate (in lesions initially managed endoscopically) of $5.7 \%$ - no residual cancer was detected following definitive treatment.

Surgery was the initial therapeutic modality in $7 / 40$ (17.5\%); $5 / 7$ (71.4\%) had segmental colectomy and $2 / 7$ (28.6\%) had TEM. No cancer was found in any surgically resected specimen.

Conclusion Most patients with LSCPs can be managed endoscopically with good outcomes, including a low adverse event profile and recurrence rate. A small proportion of cases may turn out to have carcinoma that was not possible to diagnose in pre-procedure biopsies and thus require further surgery. Our study supports high quality endoscopic assessment and multidisciplinary team discussion as important factors in achieving optimal patient management and has resulted in piloting a change of practise across the BCSP to minimise variation in outcomes.

Disclosure of Interest None Declared.

\section{PWE-044 CURRENT PRACTICE IN COMPLEX LOWER GI POLYPECTOMY: A UK NATIONAL SURVEY}

doi:10.1136/gutjnl-2013-304907.333

1."J Geraghty, 'P 0'Toole, 2J Anderson, 2R Valori, 'S Sarkar. 'Gastroenterology, Royal Liverpool University Hospital, Liverpoool; ${ }^{2}$ Gastroenterology, Gloucestershire Hospitals, Gloucester, UK

Introduction In recent years there have been considerable developments in polypectomy techniques. However, even among experienced colonoscopists, there remains a wide variation in practise. This survey attempts to evaluate current UK practise in more advanced polypectomy.

Methods NHS Bowel Cancer Screening Programme (BCSP) colonoscopists and BSG members were invited to complete an anonymous online questionnaire regarding their approach to large polyps.

Results Respondents: Complete responses were obtained from 268 colonoscopists practising independently in the UK. 75\% were consultant gastroenterologists, $10 \%$ surgeons and $9 \%$ nurse endoscopists. $41 \%$ did at least one session in a teaching hospital. $64 \%$ were BCSP accredited. All regions were well represented in the survey, although there were only 2 responders from Wales. Median lifetime colonoscopy experience was 3000 and typical workload was about 10 procedures per week.

Experience \& Practice: $86 \%$ felt confident to remove lesions > $2 \mathrm{~cm}$ by endoscopic mucosal resection (EMR). $27 \%$ of this group had done $<10$ EMR procedures in the previous year; $14 \%$ claimed $>50$ EMRs per year. When asked to describe the most complex polypectomy they would undertake, $30 \%$ (81) judged themselves capable of resecting very large flat or polypoid lesions that are also suitable for surgery (i.e. Level 4 polypectomy). Of these Level 4 operators, 17\% had never tackled a polyp greater than $5 \mathrm{~cm}$ and $10 \%$ had performed $<10$ EMRs in the previous year. Video recording was used in the assessment of difficult polyps by only $20 \%$. $32 \%$ declared that they would routinely biopsy a potentially resectable polyp they did not feel comfortable to remove themselves, with $3.5 \%$ opting to snare a large piece for histology.

Endoscopic Submucosal Dissection (ESD): 7 responders perform full ESD and a further 13 use ESD as part of a hybrid technique. $11 \%$ had referred a patient to another specialist for ESD in the last year.

Complications: Among those performing EMR, 10\% admitted to a perforation in the previous year and $23 \%$ reported significant bleeding.

Conclusion This is the first "snap shot" of advanced polypectomy practise across the UK. Although the sample was self-selected, a range of experience is represented. Most experienced colonoscopists appear happy to attempt piecemeal EMR even if their annual numbers are low. As expected, very few colonoscopists are performing ESD. A surprising number of colonoscopists regarded themselves as Level 4 experts, suggesting that the current definition of what constitutes very advanced practise needs to be modified, or at least better defined. The limited use of video documentation is disappointing and unhelpful practises, such as routine polyp biopsy (or partial polypectomy), are still relatively common

Disclosure of Interest J. Geraghty Grant/Research Support from: COOK MEDICAL, P. O’Toole: None Declared, J. Anderson: None Declared, R. Valori: None Declared, S. Sarkar: None Declared

\section{PWE-045 TRAINING IN ADVANCED POLYPECTOMY: RESULTS FROM A NATIONAL UK SURVEY}

doi:10.1136/gutjnl-2013-304907.334

1,"J Anderson, ${ }^{2 P}$ O'Toole, ${ }^{2} \mathrm{~J}$ Geraghty, 'R Valori, ${ }^{2} \mathrm{~S}$ Sarkar. ' Gastroenterology, Gloucestershire Hospitals, Gloucester; ' ${ }^{2}$ Gastroenterology, Royal Liverpool University Hospital, Liverpoool, UK

Introduction Despite considerable developments in polypectomy techniques, the training of the skills necessary for removal of complex polyps remains a challenge. Little is known about the level of formal training in polypectomy among colonoscopists currently practising in the UK.

Methods As part of a national survey of advanced polypectomy, targeted at BSG members and Bowel Cancer Screening Programme (BCSP) colonoscopists, individuals were asked about the training they had received in polypectomy and their perceived training needs.

Results Respondents 268 fully trained colonoscopists with a median lifetime experience of 3000 procedures. $64 \%$ were BCSP colonoscopists. All but 4 had been involved in a hands-on colonoscopy-related training course and almost half had acted as course faculty.

Competence $86 \%$ reported competence in endoscopic mucosal resection (EMR) of sessile polyps $>2 \mathrm{~cm}$ and half of responders had been doing EMR for $>5$ years. 30\% felt comfortable removing lesions up to $3 \mathrm{~cm}$ with good access, $35 \%$ would tackle larger lesions (by EMR) or smaller lesions with difficult access. $30 \%$ considered themselves able to remove very large flat or polypoid lesions that were also suitable for surgery.

Formal Training In relation to EMR, 58\% regarded themselves as predominantly self-taught. $24 \%$ had been on an attachment at a recognised training centre and $36 \%$ had attended a hands-on advanced colonoscopy or polypectomy course. $20 \%$ reported that their only formal education in EMR was at a demonstration workshop or study day.

Training Needs When asked what limited their advanced polypectomy practise, $18 \%$ identified lack of formal training in EMR, 18\% lack of opportunity to gain experience and $7.5 \%$ lack of guidelines in the management of large polyps. Surprisingly, even colonoscopists claiming to operate at the most expert level admitted that they might decide against tackling a potentially resectable polyp because of lack of confidence in assessing surface morphology (7\%). $2.5 \%$ 